\title{
THE IMPORTANCE OF $\Pi_{1}^{0}$ CLASSES IN EFFECTIVE RANDOMNESS.
}

\author{
GEORGE BARMPALIAS, ANDREW E.M. LEWIS, AND KENG MENG NG
}

\begin{abstract}
We prove a number of results in effective randomness, using methods in which $\Pi_{1}^{0}$ classes play an essential role. The results proved include the fact that every PA Turing degree is the join of two random Turing degrees, and the existence of a minimal pair of LR degrees below the LR degree of the halting problem.
\end{abstract}

\section{INTRODUCTION}

1.1. $\Pi_{1}^{0}$ classes in computability and effective randomness. Many arguments in computability theory and algorithmic randomness involve $\Pi_{1}^{0}$ sets of reals and techniques specific to such sets in an essential way. Two major references to such arguments in computability theory and in particular the degrees of unsolvability, are the well known Jockusch-Soare papers [JS72a, JS72b] on degrees of theories and members of $\Pi_{1}^{0}$ classes. In this work, Jockusch and Soare introduced the method of forcing with $\Pi_{1}^{0}$ classes, proved the now classic low basis theorem and showed a number of degree theoretic results using compactness arguments with $\Pi_{1}^{0}$ sets. For a survey of results concerning $\Pi_{1}^{0}$ classes in computability theory we refer the reader to Cenzer [Cen99]. In algorithmic randomness, Kučera's early papers [Kuč85, Kuč86] (partly inspired by some questions in the above papers of Jockusch and Soare) are a demonstration of how central $\Pi_{1}^{0}$ classes are in the study of the degrees of complete extensions of Peano Arithmetic (PA degrees) and effective randomness. In this work he also introduced fundamental methods for coding into PA degrees (using universal $\Pi_{1}^{0}$ classes) and coding into effectively random sets (using $\Pi_{1}^{0}$ classes of positive measure). The importance of $\Pi_{1}^{0}$ sets in arguments can be seen in a lot of recent work. As an example we mention the construction of a low bound for the ideal of K-trivial degrees by Kučera and Slaman [KS07] which uses $\Pi_{1}^{0}$ sets for coding in a very essential way.

In this paper we show a number of results about PA degrees and relative randomness demonstrating the applicability of methods with $\Pi_{1}^{0}$ classes to

Key words and phrases. $\Pi_{1}^{0}$ classes, Martin-Löf randomness, Peano Arithmetic, Ktrivials, Turing degrees, LR degrees.

Barmpalias was supported by the Marsden Foundation of New Zealand, via a postdoctoral fellowship. Lewis was supported by a Royal Society Research Fellowship. We would like to thank Antonio Montalbán for a helpful discussion on the proof of theorem 2.1. 
the solution of some problems in this area, which do not currently have a known solution via different methods. Firstly we show that every PA degree is the least upper bound of two Martin-Löf random degrees, thus revealing more connections between these two classes of degrees, in the spirit of Kučera's work (see background below). Secondly, we study relative randomness and in particular the LR degrees using (for the first time in the literature) methods based on $\Pi_{1}^{0}$ sets. The main result of this approach is the construction of a minimal pair of LR degrees below the LR degree of the halting problem. This was a problem in the area of relative randomness which had resisted other techniques.

1.2. Background: PA and Martin-Löf random degrees. The collection of sets separating two disjoint c.e. sets is a natural $\Pi_{1}^{0}$ class. Hence the set of complete extensions of a consistent theory forms a $\Pi_{1}^{0}$ class, which is universal in some sense. A Turing degree is called PA if it is the degree of a complete extension of Peano Arithmetic. One of the standard characterizations of the PA degrees (due to Scott and Solovay, see e.g. [Odi89]) says that a Turing degree $\mathbf{a}$ is PA iff the degrees below a form a basis for $\Pi_{1}^{0}$ classes. In [JS72a, JS72b] Jockusch and Soare, partly motivated by the study of PA degrees, demonstrated how $\Pi_{1}^{0}$ classes and compactness arguments can be used in order to prove results about the Turing degrees. One of the most popular definitions of algorithmic randomness is the one given by MartinLöf in [ML66], according to which a set is (Martin-Löf ) random $^{1}$ if it does not belong in any 'effectively null' set in the Cantor space. Effectively null sets were defined to be those of the form $\cap_{j} E_{j}$ where $\left(E_{j}\right)$ is a uniformly c.e. sequence of $\Sigma_{1}^{0}$ classes such that $\mu\left(E_{j}\right)<2^{-j-1}$.

Kučera was the first to see the strong connection between the PA degrees and the Martin-Löf random degrees. In particular, in his well known early paper [Kuč85] he demonstrated how coding techniques based on $\Pi_{1}^{0}$ classes of positive measure can be applied in order to show results about the degrees of Martin-Löf random sequences. A distinctive feature of Kučera's work has always been that the theory of Martin-Löf random degrees is developed in parallel to the theory of PA degrees, with the techniques in the two topics being intrinsically connected. A definitive result about the relation between the PA degrees and the Martin-Löf random degrees (extending previous work of Kučera) was shown by Frank Stephan in [Ste06] and says that a PA degree is Martin-Löf random iff it computes the halting problem. As discussed in [Ste06] this result strongly suggests a dichotomy of the Martin-Löf random degrees to the ones which contain a lot of information (they compute the halting problem) and the ones which are computationally weak, in the sense that they are not PA. In Section 2 we reveal another connection between these classes of degrees: every PA degree is the least upper bound of two Martin-Löf random degrees. The techniques involved in the proof are based on properties of $\Pi_{1}^{0}$ classes and $\Pi_{1}^{0}$ classes of positive measure.

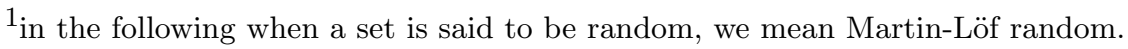


1.3. Background: relative randomness, prefix-free complexity and LR degrees. In Sections 3, 3.2 we demonstrate how methods that are based on $\Pi_{1}^{0}$ classes can be used to prove results about relative randomness. Martin-Löf randomness is equivalent to the so-called Chaitin-Levin randomness, which is based on Kolmogorov's idea of incompressibility of binary strings ${ }^{2}$. Let $K$ denote the prefix-free complexity relative to a fixed universal prefix-free machine. A set $X$ is called Chaitin-Levin random if its initial segments all have high $K$-complexity, i.e. $K(X\lceil n) \geq n-c$ for all $n \in \mathbb{N}$ and a constant $c$. A standard measure of relative compressing power of oracles is the LK reducibility $\leq_{L K}$ introduced in [Nie05b]. We say that $A \leq_{L K} B$ if the prefix-free complexity of any string relative to $B$ is less than the prefix-free complexity relative to $A$, modulo a constant: $K^{B}(\sigma) \leq^{+} K^{A}(\sigma)$ for all $\sigma \in 2^{<\omega}$, where $2^{<\omega}$ denotes the set of finite binary strings. This definition formalizes the intuitive idea of $B$ compressing more efficiently than $A$ (modulo a constant). It is clear that if $A \leq_{L K} B$ then every random set relative to $B$ is also random relative to $A$, which was denoted by $A \leq_{L R} B$ in [Nie05b]. The converse was shown by Miller in [Mil], so that the relations $\leq_{L K}, \leq_{L R}$ are identical. The equivalence classes induced by these relations are often called LR degrees. The least LR degree consists of the low for random sets (sets $A$ such that every Martin-Löf random is also Martin-Löf random relative to $A$ ), which coincide with the low for $\mathrm{K}$ sets (sets $A$ such that $K(\sigma) \leq^{+} K^{A}(\sigma)$ ) or even the K-trivial sets (sets $A$ whose prefix-free complexity is less than the prefix-free complexity of a computable sequence, modulo a constant). The equivalence of these three notions is one of the most important recent results in the area of algorithmic randomness and was shown in [Nie05b].

The structure of the LR degrees was studied in [BLS08, BLSss, Sim07] and one of the questions that was not answered with the techniques developed in these papers was about the existence of minimal pairs of LR degrees. Miller [Mil] later showed that there exist such minimal pairs by using a cardinality argument in conjunction with some properties of low for $\Omega$ sets, where $\Omega=\sum_{U(\sigma) \downarrow} 2^{-|\sigma|}$ is Chaitin's halting probability of a universal prefixfree machine $U$ (a set is low for $\Omega$ if $\Omega$ is Martin-Löf random relative to it). He actually showed that every pair of relatively 2-random sets form a minimal pair in the LR degrees.

In Section 3.1 we use arguments based on $\Pi_{1}^{0}$ classes to show that there is a minimal pair of LR degrees, LR below $\emptyset^{\prime}$. This result cannot be obtained with previously known methods, and was the main motivation for introducing Jockusch-Soare (as in [JS72a, JS72b]) type of arguments for the

\footnotetext{
${ }^{2}$ Given a prefix-free machine $M$ (a Turing machine with prefix-free domain) the prefixfree complexity of a string $\sigma$ relative to $M$ is the length of the shortest string $\tau$ such that $M(\tau)=\sigma$. There is a universal prefix-free machine, i.e. one that gives optimal descriptions to every string, modulo a constant. For more background on prefix-free complexity, see [Nie09].
} 
study of the LR degrees. ${ }^{3}$ We also get a number of useful facts about upper cone avoidance in the LR degrees in relation with $\Pi_{1}^{0}$ classes, which can be used in order to derive other results about $\leq_{L R}$. In Section 3.2 we discuss a number of other applications of Jockusch-Soare arguments to the study of the LR degrees. In Section 3.3 we discuss how the same methodology can give results about the connection between hyperarithmetical complexity and $\leq L R$.

Finally in Section 4 we give a simple proof of a result in [Bar06] on cupping with random sets. The first result in this topic was given in Nies [Nie07] and was later simplified by Hirschfeldt and Miller using a more general argument with $\Pi_{2}^{0}$ null classes. We use the actual construction of Hirschfeldt and Miller as a 'black box' to give a very short proof of the result in [Bar06], which was originally given as a generalization of the non-cupping result of Nies [Bar06].

\section{PA AND RANDOM TURING DEgREES}

In a number of widely cited papers [Kuč85, Kuč86] Kučera developed some theory about the PA, the dnr and the Martin-Löf random Turing degrees. In particular, he treated these classes of degrees using similar approaches, while often commenting on the differences between the coding methods available for the PA degrees and the Martin-Löf random degrees. Coding into PA degrees was seen to be much more flexible than coding into a Martin-Löf random degree, and this is also reflected in [KS07] where all the K-trivial degrees are coded into a low PA degree, while it is not known whether there is a low random degree with this property. The relation between the PA degrees and the Martin-Löf random degrees was clarified by the following result of Stephan: a PA degree is Martin-Löf random iff it computes the halting problem. This is a clear demonstration of a well-known dichotomy in the Martin-Löf random degrees. There is a sharp qualitative distinction between the complete Martin-Löf random degrees and the incomplete ones. The former are random because they have a lot of information (indeed all complete degrees are random [Kuč85]) while the latter are often branded the true Martin-Löf random degrees (see [DHNT06]) and they have, as a matter of fact, low information content. For example, by the above mentioned theorem of Stephan, they cannot compute any complete extension of Peano arithmetic.

In this section we provide a further relation between these two classes.

Theorem 2.1. Every PA degree is the join of two random degrees.

This result, combined with the above mentioned theorem of Stephan, gives a plethora of pairs of Martin-Löf random degrees which join to a degree

\footnotetext{
3 these include the compactness arguments they used, for example, in their proof that every $\Pi_{1}^{0}$ class with no computable paths contains two paths which form a minimal pair in the Turing degrees.
} 

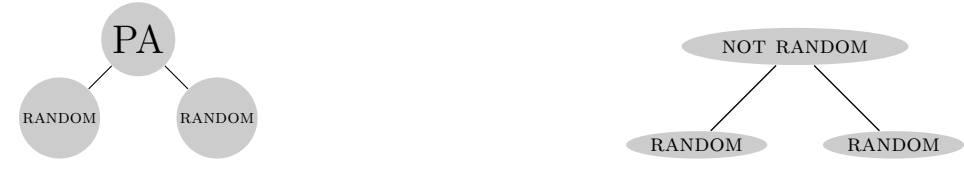

Figure 1. Every PA Turing degree is the least upper bound of two random Turing degrees. Incomplete PA Turing degrees are non-random degrees which are joins of two random degrees.

which is not Martin-Löf random; we just need to apply the result to any incomplete PA degree and get a pair with this property. In fact, this was the original motivation for this result.

Corollary 2.2. Incomplete PA degrees are non-random degrees which are the join of two random degrees.

The category version of theorem 2.1 is not true, however. Recall that a sequence is weakly 1-generic if every dense $\Sigma_{1}^{0}$ set of strings contains a prefix of it, and is 1-generic if for every $\Sigma_{1}^{0}$ set of strings which does not contain a prefix of it there is a prefix of the sequence which is not a prefix of any string in that set.

Proposition 2.3. There exists a PA degree which is not the join of two (weakly) 1-generic degrees.

Proof. By the hyperimmune-free basis theorem applied to the complete $\Pi_{1}^{0}$ class containing only complete extensions of PA, there is a hyperimmunefree PA degree. This cannot be the join of two (weakly) 1-generics as such sequences are hyperimmune by a result of Kurtz [Kur81] (also presented in [DH09]) and hyperimmune degrees are upward closed.

2.1. Introduction to the proof of Theorem 2.1. Let $C$ be a set of PA degree. We wish to find randoms $A, B$ such that $C \equiv_{T} A \oplus B$. We would like to start with a $\Pi_{1}^{0}$ class $P$ of randoms and find $A, B$ inside $P$. The plan is to use a perfect tree $T$ of paths in $P$ in order to code $C$ into the join of two of its paths $A, B$, thus achieving $C \leq_{T} A \oplus B$. The coding will be done in such a way that if $C$ can compute the tree $T$, then $A \oplus B \leq_{T} C$. In order to achieve this, we will define a class of trees $T$ such that $[T] \subseteq P$, which can be defined by a $\Pi_{1}^{0}$ formula. In other words, there is a $\Pi_{1}^{0}$ set of reals which effectively represent the trees in this class. Then we can use the fact that $C$ is of PA degree to get a tree in that class which is computable from $C$, and then use it in order to do the coding for $C \leq_{T} A \oplus B$. Before we go into the details of the argument, let us give a formal definition of a tree.

Definition 2.4. If $T$ is a partial function from $2^{<\omega}$ to $2^{<\omega}$ we say that $T$ is a tree if for every $\sigma \in 2^{<\omega}$ and $i \in\{0,1\}$ such that $T(\sigma * i) \downarrow$ : 
- $T(\sigma) \downarrow \subset T(\sigma * i)$;

- $T(\sigma *(1-i)) \downarrow \mid T(\sigma * i)$.

A tree $T$ is perfect if $T(\sigma) \downarrow$ for all $\sigma$. A finite tree $T$ of level $n$ is the restriction of a tree (as a map) to strings of length $n$.

A first attempt would be (and indeed was) to use an (infinite) indifferent set on a random sequence (with respect to the Martin-Löf random sequences) in order to get the required tree of randoms for coding (see figure 2). In [FMN] a set of positions was called indifferent for a sequence with respect to a class $\mathcal{A}$ if any alteration of the digits of the real in these positions produces a sequence in $\mathcal{A}$. It was proved (also see [Nie09]) that every random sequence has an indifferent set (with respect to the Martin-Löf random sequences). To find randoms $A, B$ such that $C \leq_{T} A \oplus B$ we can take any random $X$ and define

- $A$ to be the random we get if we let the digit in the $n$th indifferent position of $X$ be $C(n)$

- $B$ to be the random we get if we let the digit in the $n$th indifferent position of $X$ be $1-C(n)$.

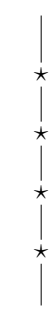

(a) Random real

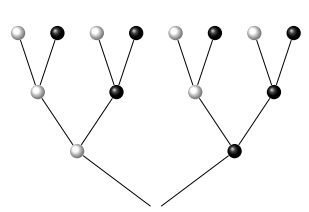

(b) Tree of random reals

FiguRE 2. (a) Indifferent set of positions $\star$ in a random sequence. (b) The associated tree of random sequences that we get if we put 0 (represented by a white ball) or 1 (represented by a black ball) into the indifferent positions on the real.

Now the indifferent set is computable in $A \oplus B$ (as it consists of the positions where $A, B$ differ) and so is $C$. However the class of trees of this type cannot be expressed as a $\Pi_{1}^{0}$ class in the Cantor space. The reason for this is that $2^{\omega}$ is compact while the space of perfect trees of definition 2.4 (even the restricted class of figure 2) with the natural topology generated by the finite trees $^{4}$, is not. So although we can achieve $C \leq_{T} A \oplus B$ we cannot achieve Turing equivalence through this approach. The solution is to work in a compact subspace of the space of trees.

\footnotetext{
${ }^{4}$ In this topology the basic open sets are indexed by the finite trees $F$ of definition 2.4 and the basic open set corresponding to a particular $F$ is the collection of perfect trees $T$ which have $F$ as an initial segment.
} 
Definition 2.5. Let $f: \mathbb{N} \rightarrow \mathbb{N}$ be an increasing function. The function $f$ defines a partition on any given infinite string $A$. Let $\left(\sigma_{A}(i)\right)$ be the unique sequence of strings such that $\left|\sigma_{A}(0)\right|=f(0),\left|\sigma_{A}(i+1)\right|=f(i+1)-f(i)$ and

$$
A=\sigma_{A}(0) * \sigma_{A}(1) * \ldots
$$

Say that $A, B$ are piecewise $f$-different from level $n$ if $\sigma_{A}(i) \neq \sigma_{B}(i)$ for all $i \geq n$.

Now given $A, B$ which are piecewise $f$-different from level $n$, define the tree $T_{A B}^{f, n}$ as follows (for convenience we let $f(-1)=0$ ):

$$
\begin{aligned}
T_{A B}^{f, n}(\emptyset) & =A \uparrow f(n-1) \\
T_{A B}^{f, n}(\tau * 0) & =T_{A B}^{f, n}(\tau) * \min \left\{\sigma_{A}(n+|\tau|), \sigma_{B}(n+|\tau|)\right\} \\
T_{A B}^{f, n}(\tau * 1) & =T_{A B}^{f, n}(\tau) * \max \left\{\sigma_{A}(n+|\tau|), \sigma_{B}(n+|\tau|)\right\}
\end{aligned}
$$

and consider the space

$$
\mathcal{T}^{f, n}=\left\{T_{A B}^{f, n} \mid A, B \text { are piecewise } f \text {-different from level } n\right\} .
$$

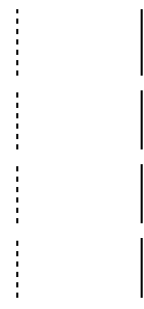

(a)

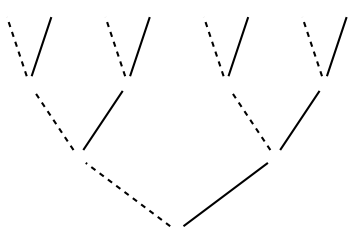

(b)

Figure 3. (a) The segments of piecewise $f$-different from level $n$ reals $A, B$. Solid and dashed lines represent lexicographically larger and smaller corresponding segments respectively. (b) The tree $T_{A B}^{f, n}$.

Now it is not hard to see that $\mathcal{T}^{f, n}$ is compact and $f$-effectively homeomorphic to the Cantor space. If $f$ is computable then the set

$\mathcal{C}_{f}^{n}=\left\{A \oplus B \mid A, B\right.$ are piecewise $f$-different from level $n$ and $\left.\left[T_{A B}^{f, n}\right] \subseteq P\right\}$

(where $P$ is a $\Pi_{1}^{0}$ class consisting entirely of random sequences) is $\Pi_{1}^{0}$ which is exactly what we wanted. It suffices to show the following.

Lemma 2.6. There exists a computable function $f$ such that, if $P$ is a $\Pi_{1}^{0}$ class of positive measure, then $\mathcal{C}_{f}^{n} \neq \emptyset$ for some $n \in \mathbb{N}$.

We need to work with finite approximations to the notion of piecewise different pairs of reals, therefore we introduce the following terminology. 
Definition 2.7 (Switching reals inside $\Pi_{1}^{0}$ classes). Let $f: \mathbb{N} \rightarrow \mathbb{N}$ be an increasing function and $P$ a $\Pi_{1}^{0}$ class. We say that $A$ can be $f$-switched (or just switched) at $[n, m]$ inside $P$ if it belongs to $P$ and there exists $B$ such that $\sigma_{A}(i) \neq \sigma_{B}(i)$ for all $i \in[n, m]$ and for every sequence $\left(x_{i}\right) \in\{A, B\}^{\omega}$ with $x_{i}=A$ for $i \notin[n, m]$, the real $\sigma_{x_{0}}(0) * \sigma_{x_{1}}(1) * \ldots$ is in $P$. In this case $B$ is a switching partner of $A$ at $[n, m]$ inside $P$. We say that $A$ can be $f$-switched (or just switched) from level $n$ inside $P$ if it belongs to $P$ and there is $B$ such that $\sigma_{A}(i) \neq \sigma_{B}(i)$ for all $i \geq n$ and for every sequence $\left(x_{i}\right) \in\{A, B\}^{\omega}$ with $x_{i}=A$ for $i<n$, the real $\sigma_{x_{0}}(0) * \sigma_{x_{1}}(1) * \ldots$ belongs to $P$.

If $C_{n, m}(A)$ denotes the class of switching partners of $A$ at $[n, m]$ inside $P$ then $C_{n}(A)=\cap_{m} C_{n, m}(A)$ is the class of switching partners of $A$ from level $n$ inside $P$. Note that $C_{n, m}(A)$ is clopen and $C_{n}(A)$ is closed. Let $D_{n, m}$ (for $0 \leq n \leq m$ ) denote the set of reals which cannot be switched at $[n, m]$ inside $P$. So $D_{n, m} \subseteq D_{n, m+1}$ and it is clear that the classes $D_{n, m}$ are $\Sigma_{1}^{0}$ uniformly in $f$. Let $D_{n}$ be the set of reals which cannot be switched from level $n$ inside $P$.

Lemma 2.8. $D_{n}=\cup_{m} D_{n, m}$ for all $n$.

Proof. It is enough to show that $\bar{D}_{n}=\cap_{m} \bar{D}_{n, m}$ for all $n$. Indeed, if $A \in$ $\cap_{m} \bar{D}_{n, m}$ by compactness we have that $C_{n}(A)=\cap_{m} C_{n, m}(A) \neq \emptyset$ and so $A \in \bar{D}_{n}$ (the other direction is trivial).

We are going to show the following, which is stronger than lemma 2.6 since, by [Kuč85], a $\Pi_{1}^{0}$ class of positive measure contains a final segment of every Martin-Löf random real.

Lemma 2.9. There exists a computable function $f$ such that if $P$ is a $\Pi_{1}^{0}$ class and $X \in P$ is sufficiently random (weakly 2-random suffices) then for some $n \in \mathbb{N}$ and some $Y$ piecewise $f$-different to $X$ from level $n$ we have $\left[T_{X Y}^{f, n}\right] \subseteq P$.

2.2. Proof of Lemma 2.9. It suffices to inductively define a computable function $f$ such that

$$
\mu\left(\hat{D}_{n}\right) \leq \mathcal{O}\left(2^{-n}\right), \text { where } \hat{D}_{n}=D_{n} \cap P .
$$

Indeed, in that case the class $\cap_{i} \hat{D}_{i}$ is $\Pi_{2}^{0}$ and is null. So for every weakly 2random $X \in P$ there some $n$ such that $X \notin D_{n}$, which means that $\left[T_{X Y}^{f, n}\right] \subseteq$ $P$ for some $Y$. For (2.2) it suffices to make

$$
\begin{aligned}
\mu\left(\hat{D}_{n, n}\right) & \leq 2^{-n-1} \\
\mu\left(\hat{D}_{n, m+1}-\hat{D}_{n, m}\right) & \leq 2^{-n-m-2}
\end{aligned}
$$

for all $n$ and all $m \geq n$, where $\hat{D}_{n, m}=P \cap D_{n, m}$. We show that

$$
\begin{aligned}
\mu\left(\hat{D}_{n, n}\right) & \leq 2^{f(n-1)} \cdot 2^{-f(n)} \\
\mu\left(\hat{D}_{n, m+1}-\hat{D}_{n, m}\right) & \leq 2^{f(m)} \cdot 2^{f(m)-f(n-1)} \cdot 2^{-f(m+1)}
\end{aligned}
$$


for an arbitrary increasing $f$, and then choose a computable $f$ appropriately. For (2.5), fix $\sigma$ of length $f(n-1)$ and for each $\tau$ of length $f(n)-f(n-1)$ let $M_{\sigma \tau}(n, n)$ be the set of reals $B$ such that $\sigma * \tau * B \in \hat{D}_{n, n}$. By the definition of $\hat{D}_{n, n}$ we have that $M_{\sigma \tau}(n, n) \cap M_{\sigma \rho}(n, n)=\emptyset$ for any strings $\tau \neq \rho$ of length $f(n)-f(n-1)$. Hence $\sum_{\tau \in 2^{f(n)-f(n-1)}} \mu\left(M_{\sigma \tau}(n, n)\right) \leq 1$ and so $\mu\left(\hat{D}_{n, n} \cap[\sigma]\right) \leq 2^{-f(n)}$. Given that there are $2^{f(n-1)}$ such strings $\sigma$, we get (2.5).

For (2.6), say that a string $\tau$ of length $f(m)-f(n-1)$ is a switching string for $A \in P$ at $[n, m]$ inside $P$ if for some (equivalently, for all) $\eta$ of length $f(n-1)$ and $B \in 2^{\omega}$ the real $\eta * \tau * B$ is a switching partner for $A$ at $[n, m]$ inside $P$. For any string $\tau$ of length $f(m)-f(n-1)$ let $L_{\tau}$ be the set of reals in $P$ for which $\tau$ is a switching string at $[n, m]$. Since every $A \in P-\hat{D}_{n, m}$ belongs to some $L_{\tau}$ we have

$$
\hat{D}_{n, m+1}-\hat{D}_{n, m}=\cup\left\{\hat{D}_{n, m+1} \cap L_{\tau} \cap[\sigma] \mid \sigma \in 2^{f(m)} \wedge \tau \in 2^{f(m)-f(n-1)}\right\} .
$$

Fix strings $\sigma, \tau$ of lengths as in (2.7) and for each string $\rho$ of length $f(m+1)-$ $f(m)$ let $M_{\sigma \rho, \tau}(n, m+1)$ be the set of all $B$ such that $\sigma * \rho * B \in \hat{D}_{n, m+1} \cap L_{\tau}$. As before we have that $M_{\sigma \rho, \tau}(n, m+1) \cap M_{\sigma \rho^{\prime}, \tau}(n, m+1)=\emptyset$ for any $\rho \neq \rho^{\prime}$ of length $f(m+1)-f(m)$. Hence

$$
\sum_{\rho \in 2^{f(m+1)-f(m)}} \mu\left(M_{\sigma \rho, \tau}(n, m+1)\right) \leq 1
$$

and so $\mu\left(\hat{D}_{n, m+1} \cap L_{\tau} \cap[\sigma]\right) \leq 2^{-f(m+1)}$. Then from (2.7) we get (2.6). Now if we let

$$
f(0)=1 \quad f(n+1)=2 f(n)+n+2
$$

then (2.5) and (2.6) give (2.3) and (2.4) respectively. This concludes the proof of the lemma.

2.3. Proof of Theorem 2.1. Let $P$ be a $\Pi_{1}^{0}$ class which contains only Martin-Löf random reals, fix $f$ as defined in (2.8) and consider $n$ such that $\mathcal{C}_{f}^{n} \neq \emptyset$. If $C$ is of $P A$ degree it computes some member $T_{A B}^{f, n}$ of $\mathcal{C}_{f}^{n}$. Without loss of generality we can assume that $A\lceil f(n-1)=B\lceil f(n-1)$ and that for each $i \geq n$ the string $\sigma_{A}(i)$ is lexicographically smaller than $\sigma_{B}(i)$ iff $C(i)=0$. Then $A, B \in P$ and so they are random reals. We claim that $C \equiv_{T} A \oplus B$. It is clear that $A \leq_{T} C$ and $B \leq_{T} C$. On the other hand for every $i, C(i)=0$ iff $\sigma_{A}(i)$ is lexicographically smaller than $\sigma_{B}(i)$.

\section{3. $L R$ minimal pairs And JockUsch-SoARE ARGuments with $\Pi_{1}^{0}$ CLASSES}

3.1. LR minimal pairs and $\Pi_{1}^{0}$ classes. A basic question that one can ask about a reducibility on $2^{\omega}$ or a degree structure is whether there exist minimal pairs. These are pairs of non-trivial (with respect to the particular reducibility) reals with the property that every real below both of them is trivial. Minimal pairs usually (for example with respect to the Turing 
reducibility) formalize the basic idea that two reals have no non-trivial common information. The same intuition applies to other reducibilities like LK, only that the notion of triviality is now weaker: a real is trivial with respect to $\leq_{L K}$ if it does not help a prefix free machine to compress more efficiently than when it works without an oracle. A minimal pair with respect to $\leq_{L K}$ is a pair of reals such that for every non-trivial real $X$ at least one of them does not compress more efficiently than $X$.

Minimal pairs of LR (and so, LK) degrees were first constructed by Miller [Mil] via a measure theoretic argument (this proof also appears in [Nie09, Exercise 8.1.12]). He showed that for every low for $\Omega$ real $A$ the set $\left\{X \mid X \leq_{L R} A\right\}$ is countable hence, given that non-trivial LR upper cones are null, he deduced the claim by the fact that a countable union of null sets is null. ${ }^{5}$ With a similar argument (see [Mil, Nie09]) he also showed that every pair of relatively 2-random reals form a minimal pair in the LR degrees (this was also observed by $\mathrm{Yu}$ Liang). Since there is a pair of $\mathbf{0}^{\prime \prime}$-computable relatively 2-random reals ${ }^{6}$, this implies that there two reals Turing below $\mathbf{0}^{\prime \prime}$ which form a minimal pair in the LR degrees.

In this section we present another method of obtaining minimal pairs of LR degrees, which allows to construct them inside null sets, for example LR below $\emptyset^{\prime}$. This methodology involves $\Pi_{1}^{0}$ sets of reals and compactness arguments and goes back to [JS72b, JS72a] where it was used to derive results about the Turing degrees. Before we start the formal argument we recall the characterization of $\leq_{L R}$ given in [KH07]: $A \leq_{L R} B$ iff there exists $V^{B}$ which is a $\Sigma_{1}^{0}(B)$ class of measure $<1$ such that $U^{A} \subseteq V^{B}$ where $U^{A}$ is a member of the universal Martin-Löf test relative to $A$; also, $A \leq_{L R} B$ iff every $\Sigma_{1}^{0}(A)$ class of measure $<1$ is contained in a $\Sigma_{1}^{0}(B)$ class of measure $<1$. From now on $U$ will always denote a fixed member of the universal oracle Martin-Löf test and the term bounded $\Sigma_{1}^{0}(X)$ will refer to a $\Sigma_{1}^{0}(X)$ class of measure $<1$. We often use the term oracle $\Sigma_{1}^{0}$ class to refer to a c.e. operator which takes a set $X$ to an $X$-c.e. set $W^{X}$ of strings, which is seen as the $\Sigma_{1}^{0}(X)$ class of reals consisting of the infinite binary extensions of the strings in $W^{X}$.

The following is an atomic version of LR cone avoidance inside a $\Pi_{1}^{0}$ class.

Lemma 3.1. Let $P$ be a nonempty $\Pi_{1}^{0}$ class, $V$ an oracle $\Sigma_{1}^{0}$ class such that $\forall Z \in 2^{\omega}, \mu\left(V^{Z}\right)<1$, and $A \mathbb{L}_{L R} \emptyset$. Then there exists some $B \in P$ such that $U^{A} \nsubseteq V^{B}$.

Proof. Suppose that for all $B \in P$ we have $U^{A} \subseteq V^{B}$. We define a $\Sigma_{1}^{0}$ class $E$ such that $\mu(E)<1$ and $U^{A} \subseteq E$, which shows that $A \leq_{L R} \emptyset$. Let

$$
E=\left\{\sigma \mid[\sigma] \subseteq V^{Z} \text { for all } Z \in P\right\} .
$$

\footnotetext{
${ }^{5}$ The same argument gives minimal pairs in the Turing degrees, but in that case it is much easier since all Turing lower cones are countable.

${ }^{6}$ This follows by the low basis theorem relativised to $\emptyset^{\prime}$ and the existence of a $\Pi_{1}^{0}\left(\emptyset^{\prime}\right)$ class which contains only 2-randoms.
} 
By hypothesis we have $U^{A} \subseteq E$ and by compactness $E$ is a $\Sigma_{1}^{0}$ class. Now take $Z \in P$ which exists since $P \neq \emptyset$. Then $E \subseteq V^{Z}$ and hence $\mu(E) \leq$ $\mu\left(V^{Z}\right)<1$.

Now we are ready to show the full LR cone avoidance theorem inside a $\Pi_{1}^{0}$ class.

Theorem 3.2. Given a nonempty $\Pi_{1}^{0}$ class $P$ and a countable sequence $\left(C_{i}\right)$ of sets such that $C_{i} \mathbb{Z}_{L R} \emptyset$ for all $i \in \mathbb{N}$, there exists $B \in P$ such that $C_{i} \not_{L R} B$ for all $i$.

Proof. We show this for one $C$ as it is clear how to generalize to $\left(C_{i}\right)$. Let $\left(V_{e}\right)$ be an effective enumeration of all oracle $\Sigma_{1}^{0}$ classes of bounded measure. We force with $\Pi_{1}^{0}$ classes and use lemma 3.1. First note that if $U^{A} \nsubseteq V_{e}^{Z}$ then by the compactness of computations ${ }^{7}$ there is $n \in \mathbb{N}$ such that $U^{A\lceil n} \nsubseteq V_{e}^{Z}$. So if $Q_{e}$ is a $\Pi_{1}^{0}$ class containing $Z$ such that $U^{A} \nsubseteq V_{e}^{Z}$ then there is some $n \in \mathbb{N}$ such that the $\Pi_{1}^{0}$ subclass

$$
M_{e, n}=\left\{Z \in Q_{e} \mid U^{A\lceil n} \nsubseteq V_{e}^{Z}\right\}
$$

is nonempty. Now let $P_{0}=P$ and inductively suppose that $P_{i} \downarrow$ and $P_{i} \neq \emptyset$. By lemma 3.1 and the previous discussion there is $n_{i} \in \mathbb{N}$ such that the $\Pi_{1}^{0}$ class

$$
\left\{Z \in P_{i} \mid U^{C \mid n_{i}} \not \subset V_{i}^{Z}\right\}
$$

is nonempty. Let $P_{i+1}$ be this class. Now if we take $B \in \cap_{i} P_{i}$, then by construction we have $B \in P$ and $U^{C} \nsubseteq V_{i}^{B}$ for all $i \in \mathbb{N}$.

An application of Theorem 3.2 gives the following, in analogy with the line of argument in [JS72b] for the Turing degrees.

Theorem 3.3. Every nonempty $\Pi_{1}^{0}$ class contains two paths with greatest lower bound 0 in the LR degrees.

Proof. Let $P$ be a nonempty $\Pi_{1}^{0}$ class. If it contains a low for random path, the claim is immediate. Otherwise, by the low for $\Omega$ basis theorem of [DHMN05] pick $C \in P$ which is low for $\Omega$. By [Mil] the lower cone

$$
\left\{Z \mid Z \leq_{L R} C\right\}
$$

is countable, so let $\left(C_{i}\right)$ be an enumeration of it. Now apply Theorem 3.2 and get $B \in P$ such that $C_{i} \not \underline{L}_{L R} B$ for all $i \in \mathbb{N}$. Then $B, C$ form a minimal pair in the $L R$ degrees.

Now a combination of Theorem 3.3 with a result from [BLSss] gives the minimal pair LR below $\emptyset^{\prime}$ that we mentioned above.

Corollary 3.4. There are $A, B \leq_{L R} \emptyset^{\prime}$ which form a minimal pair in the $L R$ degrees.

\footnotetext{
$7_{\text {that }}$ is, the fact that oracle computations only use a finite segment of the oracle. This is also known as the use principle.
} 
Proof. In [BLSss] it is shown that there is $\Pi_{1}^{0}$ class $P$ such that $Z \leq_{L R} \emptyset^{\prime}$ and $Z \mathbb{Z}_{L R} \emptyset$ for all $Z \in P$. If we apply Theorem 3.3 to $P$ we get the claim.

All known minimal pairs in the LR degrees have the property that one member of the pair has countable lower cone and, indeed, is low for $\Omega .^{8}$ It is therefore natural to ask the following.

Question 3.5. Are there sets $A, B$ which have uncountable LR lower cones and form a minimal pair in the LR degrees?

3.2. More Jockusch-Soare arguments with $\leq_{L R}$. The methodology introduced in [JS72a, JS72b] for the study of the Turing degrees through $\Pi_{1}^{0}$ classes and compactness arguments can also be applied to the study of randomness (weak) reducibilities - for example, the study of $\leq_{L R}$. We give a number of examples of such an approach, omitting proofs in the interest of space. ${ }^{9}$ Recall that the degree spectrum (with respect to some notion of degrees) of a $\Pi_{1}^{0}$ class is the set of the degrees of its members. We can show that the LR degree spectrum of a $\Pi_{1}^{0}$ class with no K-trivial members contains an antichain of size $2^{\aleph_{0}}$. Moreover this antichain can be chosen disjoint from any given countable sequence of non-trivial LR upper cones. This can be seen as an analog for $\leq_{L R}$ of Theorem 2.5 in [JS72b] which referred to the Turing degrees. Notice that a collection of reals that form an antichain of LR degrees also forms an antichain in the Turing degrees. In connection to this result, it is well known (see Sacks [Sac63]) that the Turing degree spectrum of every perfect set of reals contains an antichain of size $2^{\aleph_{0}}$. We do not know if the same holds for the LR degrees.

In relation to category, we can show that the $L R$ upper closure of a $\Pi_{1}^{0}$ class which does not contain K-trivials is meager. This can be seen as the LR analogue of Theorem 5.1 in [JS72b]. The latter result states that the Turing upper closure of a $\Pi_{1}^{0}$ class with no computable members is meager. Naturally, results about the spectra of $\Pi_{1}^{0}$ classes have consequences in the study of the Medvedev and Muchnik lattices of $\Pi_{1}^{0}$ classes. Motivated by these connections, Cole and Simpson showed in [CS07] that given any special $\Pi_{1}^{0}$ class $P$ (i.e. one containing no computable paths) we can find another special nonempty $\Pi_{1}^{0}$ class $Q$ such that $X \not_{T} Y$ for all $X \in P, Y \in Q$. The analog of this result for the LR degrees is also true: given a $\Pi_{1}^{0}$ class containing no K-trivials we can find another nonempty $\Pi_{1}^{0}$ class $Q$ containing no K-trivials, such that $X \mathbb{L}_{L R} Y$ for all $X \in P, Y \in Q$. Since every $\Pi_{1}^{0}$ class contains a path of c.e. Turing degree, this implies that: if $P$ is a $\Pi_{1}^{0}$ class with no K-trivial members then there exists a c.e. set $A$ which is not $K$-trivial and $X \mathbb{L}_{L R} A$ for all $X \in P$. Finally, we can show the

\footnotetext{
${ }^{8}$ by definition the property low for $\Omega$ is downward closed with respect to $\leq_{L R}$.

${ }^{9}$ The proofs for the results presented in this Sections 3.2 and 3.3 were removed from an earlier version of the paper, after a request by the referee. They are available from the authors.
} 
following, which can be seen as a strengthening of Theorem 4.7 of [JS72b]. There is a perfect $\Pi_{1}^{0}$ class such that any two distinct members of it are LR incomparable.

3.3. Hyperarithmetical complexity LR below $\emptyset^{\prime}$. The Jockusch-Soare methodology discussed above is also a very useful tool for the study of features of $\leq_{L R}$ that do not have an analogue in the Turing degrees. For example, we can show that there is a proper hyperarithmetical hierarchy of LR degrees below the LR degree of the halting problem. An LR degree is $\Delta_{2}^{0}$ if it contains a $\Delta_{2}^{0}$ set. Similarly, it is $\Delta_{\alpha}^{0}$ (where $\alpha$ is a computable ordinal) if it contains a set in $\Delta_{\alpha}^{0}$. For the definition of the hyperarithmetical hierarchy we refer the reader to [AK00]. Recall from [AK00] that given Kleene's $\mathcal{O}$ as a system of notations for the computable ordinals we can define the sets $H(a)$ for $a \in \mathcal{O}$ by recursion, in such way that $H(x) \equiv_{T} H(y)$ for notations $x, y \in \mathcal{O}$ representing the same ordinal. Given a computable ordinal $\alpha$, let $\emptyset^{(\alpha)}$ be some $H(a)$ for a notation $a \in \mathcal{O}$ such that $a$ represents $\alpha$. For an infinite ordinal $\alpha$ we let $\Delta_{\alpha}^{0}$ be the class of oracles which are computable from $\emptyset^{(\alpha)}$; for finite ordinals $n$ let $\Delta_{n}^{0}$ be the usual arithmetical class $\Sigma_{n}^{0} \cap \Pi_{n}^{0}$ (notice the non-uniformity in the transition from the finite to the infinite case). We can show that for each computable ordinal $\alpha \geq 2$ there is an LR degree below the LR degree of $\emptyset^{\prime}$, which is $\Delta_{\alpha}^{0}$ and is not $\Delta_{\gamma}^{0}$ for any $\gamma<\alpha$. The proof is a forcing argument with $\Pi_{1}^{0}$ classes. It uses a $\Pi_{1}^{0}$ class with no K-trivial paths, such that all of its paths are $\leq_{L R} \emptyset^{\prime}$. This was constructed in [BLSss]. Also, it uses certain features of $\leq_{L R}$ like those used in Section 3.1 .

\section{RANDOM NON-CUPPING REVISITED.}

The property of joining a random degree to $\emptyset^{\prime}$ was first addressed by Kučera in a meeting in Cordoba in 2004. The first result was produced by Nies in [Nie07] where he constructed a promptly simple set which cannot be joined with a random to $\emptyset^{\prime}$. Such sets with the latter property were called Martin-Löf non-cuppable. He also observed that such a set has to be K-trivial and showed that the above result holds even if we replace $\emptyset^{\prime}$ with an arbitrary $\Delta_{2}^{0}$ random set. Shortly after this proof was circulated, Barmpalias [Bar06] produced a different proof, which shows the stronger result that $\emptyset^{\prime}$ in the above statement can be replaced by an arbitrary $\Delta_{2}^{0}$ set. In fact, he showed the following.

Theorem 4.1 (Barmpalias [Bar06]). For every $\Delta_{2}^{0}$ noncomputable set $Y$ there is a promptly simple set $A$ such that $Y \leq_{T} A \oplus R \Rightarrow A \leq_{T} R$ for every random set $R$.

Later Hirschfeldt showed that if $\emptyset^{\prime} \leq_{T} A \oplus R$ for a K-trivial $A$ and a Martin-Löf random set $R$ then $\emptyset^{\prime} \leq_{L R} R$, and Miller and Hirschfeldt (see [Nie05a] or [Nie09]) showed that for every null $\Sigma_{3}^{0}$ class there exists a promptly simple set which is Turing below all Martin-Löf random members 
of the class. Given that the class of LR-complete sets is $\Sigma_{3}^{0}$ and null, and it contains Turing incomplete Martin-Löf random sets, it follows that there is a promptly simple Martin-Löf non-cuppable set.

Let $\left(W_{e}\right)$ be the standard effective sequence of all c.e. sets and let $\left(\Phi_{e}\right)$ be the standard effective sequence of all Turing functionals. In the following we give a simple proof of Theorem 4.1 given the proof of the above mentioned result of Hirschfeldt and Miller. Their proof is effective and produces a c.e. set. It can also easily be combined with lowness requirements, so that it uniformly produces a lowness index for the constructed set. In other words, this modified argument can be seen as a pair of computable functions $f_{1}, f_{2}$ which take an index of a $\Sigma_{3}^{0} \operatorname{class}^{10} S$ and return a c.e. index $f_{1}(e)$ of a c.e. set $W$ (i.e. a number $k$ such that $\left.W=W_{k}\right)$ and a lowness index $f_{2}(e)$ of $W$ (i.e. a number $t$ such that $W^{\prime}=\Phi_{t}^{\emptyset^{\prime}}$ ) such that

- $W$ is Turing below all Martin-Löf random members of $S$

- if $S$ is null then $W$ is promptly simple.

Notice that by the properties of $f_{1}, f_{2}$ we also have $W_{f_{1}(e)}^{\prime}=\Phi_{f_{2}(e)}^{\emptyset^{\prime}}$ for all $e$. Given a non-computable $\Delta_{2}^{0}$ set $Y$ with a computable approximation $\left(Y_{s}\right)$ the class

$$
S_{e}=\left\{X \mid Y \leq_{T} X \oplus W_{e}\right\}=\cup_{m} \cap_{s_{0}, n} \cup_{s>s_{0}}\left\{X \mid \Phi_{m, s}^{X \oplus W_{e}} \supset Y_{s} \uparrow n\right\}
$$

(where $\Phi_{m, s}$ denotes the finite approximation to the Turing functional $\Phi_{m}$ at stage $s)$ is $\Sigma_{3}^{0}\left(W_{e}\right)$. Also, $S_{e}$ is null in the case that $Y \mathbb{Z}_{T} W_{e}$, and it is $\Sigma_{3}^{0}$ in the special case where $W_{e}$ is low. In fact, there is a computable function $g$ such that if $n$ is a lowness index of a low set $W_{e}$ then $g(e, n)$ is a $\Sigma_{3}^{0}$ index of $S_{e}$. By the double recursion theorem there exist $e, k \in \mathbb{N}$ such that

$$
W_{f_{1}(g(e, k))}=W_{e} \text { and } \Phi_{f_{2}(g(e, k))}^{\emptyset^{\prime}}=\Phi_{k}^{\emptyset^{\prime}}=W_{e}^{\prime} .
$$

The set $W_{e}$ is not computable, because otherwise $Y \mathbb{Z}_{T} W_{e}$, so by [Sti72] $S_{e}$ is null and therefore $W_{f_{1}(g(e, k))}$ is promptly simple (by the properties of $f_{1}$ ), a contradiction. If $Y \leq_{T} W_{e} \oplus R$ for any Martin-Löf random set $R$, then $R \in S_{e}$ and by the properties of $f_{1}$ we have $W_{e} \leq_{T} R$.

\section{REFERENCES}

[AK00] C. J. Ash and J. Knight. Computable structures and the hyperarithmetical hierarchy, volume 144 of Studies in Logic and the Foundations of Mathematics. North-Holland Publishing Co., Amsterdam, 2000.

[Bar06] George Barmpalias. Random non-cupping revisited. J. Complexity, 22(6):850857, 2006.

[BLS08] George Barmpalias, Andrew E. M. Lewis, and Mariya Soskova. Randomness, Lowness and Degrees. J. of Symbolic Logic, 73(2):559-577, 2008.

[BLSss] George Barmpalias, Andrew E. M. Lewis, and Frank Stephan. $\Pi_{1}^{0}$ classes, LR degrees and Turing degrees. Ann. Pure Appl. Logic, (in press).

\footnotetext{
10 as usual, we can assume that every number is the index of some $\Sigma_{3}^{0}$ class. Recall that a $\left(\Sigma_{3}^{0}\right)$ index of a $\Sigma_{3}^{0}$ class $S$ is the index of a Turing machine which, given a triple $(i, j, k)$, outputs a clopen set of reals $V_{i, j, k}$ such that $S=\cup_{i} \cap_{j} \cup_{k} V_{i, j, k}$.
} 
[Cen99] Douglas Cenzer. $\Pi_{1}^{0}$ classes in computability theory. In Handbook of computability theory, volume 140 of Stud. Logic Found. Math., pages 37-85. NorthHolland, Amsterdam, 1999.

[CS07] Joshua A. Cole and Stephen G. Simpson. Mass problems and hyperarithmeticity. J. Math. Log., 7(2):125-143, 2007.

[DH09] Rod Downey and Denis Hirshfeldt. Algorithmic Randomness and Complexity. Springer-Verlag, in preparation, 2009.

[DHMN05] Rod Downey, Denis R. Hirschfeldt, Joseph S. Miller, and André Nies. Relativizing Chaitin's halting probability. J. Math. Log., 5(2):167-192, 2005.

[DHNT06] Rod Downey, Denis R. Hirschfeldt, André Nies, and Sebastiaan A. Terwijn. Calibrating randomness. Bull. Symbolic Logic, 12(3):411-491, 2006.

[FMN] Santiago Figueira, Joseph Miller, and André Nies. Indifferent sets. To appear.

[JS72a] Carl G. Jockusch, Jr. and Robert I. Soare. Degrees of members of $\Pi_{1}^{0}$ classes. Pacific J. Math., 40:605-616, 1972.

[JS72b] Carl G. Jockusch, Jr. and Robert I. Soare. $\Pi_{1}^{0}$ classes and degrees of theories. Trans. Amer. Math. Soc., 173:33-56, 1972.

[KH07] Bjørn Kjos-Hanssen. Low for random reals and positive-measure domination. Proc. Amer. Math. Soc., 135(11):3703-3709 (electronic), 2007.

[KS07] Antonín Kučera and Theodore Slaman. Low upper bounds of ideals. Preprint, 2007.

[Kuč85] Antonín Kučera. Measure, $\Pi_{1}^{0}$-classes and complete extensions of PA. In Recursion theory week (Oberwolfach, 1984), volume 1141 of Lecture Notes in Math., pages 245-259. Springer, Berlin, 1985.

[Kuč86] Antonín Kučera. An alternative, priority-free, solution to Post's problem. In Mathematical foundations of computer science, 1986 (Bratislava, 1986), volume 233 of Lecture Notes in Comput. Sci., pages 493-500. Springer, Berlin, 1986.

[Kur81] S. Kurtz. Randomness and genericity in the degrees of unsolvability. Ph.D. Dissertation, University of Illinois, Urbana, 1981.

[Mil] Joseph S. Miller. The $K$-degrees, low for $K$ degrees, and weakly low for $K$ sets. Preprint.

[ML66] Per Martin-Löf. The definition of random sequences. Information and Control, 9:602-619, 1966.

[Nie05a] André Nies. Eliminating concepts. In Proceedings of the IMS workshop on computational aspects of infinity, Singapore, (in press), 2005.

[Nie05b] André Nies. Lowness properties and randomness. Adv. Math., 197(1):274-305, 2005.

[Nie07] André Nies. Non-cupping and randomness. Proc. Amer. Math. Soc., 135(3):837-844 (electronic), 2007.

[Nie09] André Nies. Computability and Randomness. Oxford University Press, 444 pp., 2009.

[Odi89] P. G. Odifreddi. Classical recursion theory. Vol. I. North-Holland Publishing Co., Amsterdam, 1989.

[Sac63] Gerald E. Sacks. Degrees of unsolvability. Princeton University Press, Princeton, N.J., 1963.

[Sim07] Stephen G. Simpson. Almost everywhere domination and superhighness. $M L Q$ Math. Log. Q., 53(4-5):462-482, 2007.

[Ste06] Frank Stephan. Martin-Löf random and PA-complete sets. In Logic Colloquium '02, volume 27 of Lect. Notes Log., pages 342-348. Assoc. Symbol. Logic, La Jolla, CA, 2006.

[Sti72] John Stillwell. Decidability of the "almost all" theory of degrees. J. Symbolic Logic, 37:501-506, 1972. 
George Barmpalias: School of Mathematics, Statistics, and Computer Science, Victoria University, PO Box 600 Wellington New Zealand

E-mail address: George.Barmpalias@mcs.vuw.ac.nz

$U R L:$ http://www.mcs.vuw.ac.nz/ georgeb/

Andrew E.M. Lewis: School of Mathematics, University of Leeds, Leeds LS2 9JT, U.K.

E-mail address: andy@aemlewis.com

$U R L:$ http: //www. aemlewis.com

Keng Meng Ng: School of Mathematics, Statistics, and Computer Science, Victoria University, PO Box 600 Wellington New Zealand

E-mail address: keng.meng.ng@mcs . vuw.ac.nz

$U R L:$ http://www.mcs.vuw.ac.nz/ selwyn/ 